\title{
Incidence of and Risk Factors for Tuberculosis among Cancer Patients in Endemic Area: A Regional Cohort Study
}

\author{
Sirinya Nanthanangkul ${ }^{1,2}$, Supannee Promthet ${ }^{2}$, Krittika Suwanrungruang ${ }^{2,3}$, \\ Chalongpon Santong ${ }^{3}$, Patravoot Vatanasapt ${ }^{2,3,4 *}$
}

\begin{abstract}
Background and Objective: Cancer is a known risk factor for developing active tuberculosis (TB) disease. The incidence of and risk factors for TB are not known among cancer patients in Thailand. This study aimed to investigate risk factors for TB among cancer patients in an area with endemic TB infections. Methods: We used the Khon Kaen population-based cancer registry and two TB databases to conduct a retrospective cohort study of cancer patients. From 2001 to 2015, we identified 40,948 eligible cancer patients. Following until 2017, we identified cases of TB diagnosed after cancer diagnosis and analyzed primary cancer site, staging, treatment, and demographic factors. Adjusted incidence rate ratios (adj. IRR) were computed to identify risk factors among a sub-set of cancer types $(\mathrm{n}=9,733)$ using Poisson regression. Results: Among all cancer patients, 472 cases of TB were diagnosed following cancer diagnosis (cumulative incidence $=1.15 \%$, incidence rate $=421.86$ cases per 100,000 patients per year). Among the sub-set of cancer types, 206 cases of TB were found (cumulative incidence $=2.11 \%$, incidence rate $=848.26$ cases per 100,000 patients per year). Risk factors for TB among cancer patients were sex $(\mathrm{p}<0.001)$ (male adj. IRR $=1.87,95 \%$ CI: 1.36-2.59), age $(\mathrm{p}<0.001)($ age $>70 \mathrm{adj} . \mathrm{IRR}=2.36,95 \% \mathrm{CI}: 1.56-3.55$, compared to age $\leq 50)$ and cancer site $(\mathrm{p}<0.001)$. Compared to thyroid cancer, TB infection was more associated with lung cancer without histopathological confirmation (adj. IRR $=6.22$, 95\% CI: 2.57-15.04). Cancer stage and treatment did not show statistically significant trends. Conclusion: Old age, male sex, and certain cancer types were independent risk factors for TB in cancer patients. Targeted latent TB screening may be appropriate among high risk groups.
\end{abstract}

Keywords: Epidemiology- latent infection- malignancy- screening- tuberculosis

Asian Pac J Cancer Prev, 21 (9), 2715-2721

\section{Introduction}

Cancer is a major public health problem worldwide and among the most common causes of morbidity and mortality. It has long been recognized that cancer can cause malnutrition and diminished immune functioning directly from the disease or indirectly via secondary effects. The treatment of cancer, especially chemotherapy, also plays an important role in immunosuppression. This leads to an increased risk of reactivating latent tuberculosis (TB) infections or new TB infection during the clinical course of cancer in endemic areas of the disease. Increased risk of TB infection has been found among certain cancer types, including lung, hematologic, colon, and oral (Cheng et al., 2016; Dobler et al., 2017; Huang et al., 2011; Teng et al., 2019). Thailand is among the 14 countries with the highest burden of TB, with an estimated incidence of 120,000 new cases of TB per year (WHO, 2018; Bureau of Tuberculosis, Department of Disease control Ministry of Public Health, 2017). However, the incidence of and risk factors for $\mathrm{TB}$ are not known among cancer patients in Thailand. The purpose of this study was to use a regional population-based cohort of cancer patients to analyze this issue and identify groups at higher risk of TB infection.

\section{Materials and Methods}

\section{Data Source and Study Design}

We conducted a retrospective cohort study of cancer patients. Cancer patient data was queried from the Khon Kaen population-based cancer registry. This cancer registry includes all cancer diagnoses among the population of Khon Kaen province. TB data was queried from two databases: the Khon Kaen Central Hospital TB Database, which records diagnoses of TB, and the TB database of the Region 7 Office of Disease Prevention and Control, which logs TB cases for the area of Khon Kaen province. The study was approved by the Khon

${ }^{1}$ Doctor of Philosophy Program in Epidemiology and Biostatistics, Faculty of Public Health, Khon Kaen University, Khon Kaen, Thailand. ${ }^{2}$ ASEAN Cancer Epidemiology and Prevention Research Group, Khon Kaen, Thailand. ${ }^{3}$ Cancer Unit, Srinagarind Hospital, Faculty of Medicine, Khon Kaen University, Khon Kaen, Thailand. ${ }^{4}$ Department of Otorhinolaryngology, Faculty of Medicine, Khon Kaen University, Khon Kaen, Thailand.*For Correspondence: patvat@kku.ac.th 
Kaen University Ethics Committee for Human Research (reference number HE 611542).

\section{Study Cohort}

From January 1st, 2001 to December 31st, 2015, new cancer diagnoses were identified with the International Classification of Diseases for Oncology (ICD-O 3rd edition, code $\mathrm{C} 00.0-\mathrm{C} 80.9)$ and later converted to ICD-10 for reporting $(\mathrm{n}=42,045)$. The TB databases were then queried for all TB cases (ICD-10 version 2016, code A15.0-A19.9) that were assigned to patients included in the cancer cohort (linked by ID number) between the time period from January 1st, 2001 to December 31st, 2017. After removing duplicates $(n=16,532)$, a total of 26,945 TB cases were matched to the cancer cohort (Fig. 1). Patients were excluded if the TB diagnosis was made prior to the cancer diagnosis $(n=25,945)$ or if the cancer diagnosis was not recorded until the death certificate (n $=1,097)$.

\section{Data Extraction}

The primary cancer site, staging, cancer treatment, and demographic data were extracted as independent factors. TB diagnosis and time to diagnosis were extracted as dependent variables. Cancer site was coded as one of seventeen cancer sites, including breast, colorectal, liver and bile duct, lung (with histopathological confirmation), lung (without histopathological confirmation), lymphoma, hematopoietic (leukemia), female organ (cervix, corpus, uterus, ovary, perineum), male organ (penis, prostate, testis), head and neck, skin, thyroid, digestive organ, brain, urinary tract, unknown primary and other cancers. Cancer extent was categorized according to SEER Summary Staging 2000 codes as localized, regional, distant, not applicable, or unknown. Cancer treatment included surgery, chemotherapy, radiotherapy, or combinations thereof. Demographics included sex and age.

\section{Statistical analysis}

The development of TB was the primary dependent variable for this study. Follow-up of the cohort ended on the date of TB diagnosis, the patient's death, or December 31, 2017. The cumulative incidence and incidence rate were computed using the Kaplan-Meier method. Chisquared tests were used to compare TB cases among categorical variables. Risk factors were assessed using Poisson regression analysis to compute adjusted incidence rate ratios also check assumption with the overdispersion testing then it passed this requirement. In the Poisson analysis, the dependent variable was the time to TB diagnosis following cancer or loss to follow-up, with the person-years as the risk offset variable. Factors were included in the initial model if they were found to be $\mathrm{p}$ value $<0.25$ from the bivariate analysis or if were found to be essential from reviewed literature. Only certain cancer types were included in the analysis, based on literature review that the cancer type impact to TB (thyroid, lung, lymphoma, hematopoietic, digestive organ, urinary tract cancer), yielding a final analysis sample size of 9,733. STATA statistical software (version 15.0 StataCorp. 2017. College Station, TX: StataCorp LLC) was used in the analysis.

\section{Results}

Clinical and demographic characteristics of cancer patients

From January 2001 to December 2015, 40,948 eligible patients were diagnosed with cancer and included in the study. The cohort had a median age of 60 years and had slightly more males $(51.3 \%)$ (Table 1$)$. Following until 2017, 472 post-cancer diagnoses of TB were identified (cumulative incidence $1.15 \%$ ). Post-cancer TB diagnosis was more common in males (cumulative incidence $1.41 \%$ ) and nearly equal among age groups $(\mathrm{p}=0.990)$. Cumulative incidence of post-cancer TB differed among type of cancer, with the highest proportions found in patients with lymphoma (cumulative incidence $3.05 \%$ ), hematopoietic $(2.93 \%)$ and lung cancer (without histopathological confirmation) $(2.15 \%)$ (Table 2). Considering the staging, cumulative incidence was highest among localized tumors $(1.65 \%)$, followed by regional $(1.15 \%)$, and distant metastasis $(0.93 \%)$. Among treatments, chemotherapy had the highest cumulative incidence of TB $(1.84 \%)$.

\section{Incidence rate for TB among cancer patients}

Among all cancer patients, 472 post-cancer TB cases were identified from a total of 111,886 person-years of follow-up (incidence rate 421.86 cases per 100,000 patients per year). Considering a sub-set of cancers shown in literature to be associated with higher risk of active TB infection (Table 3), 206 cases of post-cancer TB were found (cumulative incidence $=2.11 \%$ ) among 24,285 person-years of follow-up (incidence rate $=848.26$ cases per 100,000 patients per year).

\section{Risk factors for TB among cancer patients}

There was a statistically significant difference of incidence rate ratios (IRRs) among age group, gender and cancer type. All independent variables were included into the risk factor analysis. However, only a sub-set of cancer types were included (Table 3$)(n=9,733)$. The highest adjusted IRRs (adj. IRR) were for age $>70$ year (adj. IRR : 2.36, 95\% CI: 1.56-3.55), and males (adj. IRR : 1.87, 95\% CI; 1.36-2.59). For cancer types, we found lung cancer (without histopathological confirmation), lymphoma, hematopoietic, digestive organ cancer, urinary tract cancer and lung cancer (with histopathological confirmation) to have higher adj. IRR at statistically significant levels. Incidence of post-cancer TB was not statistically significantly different among cancer staging, while the trend for types of cancer treatment was unclear (Table 3).

\section{Discussion}

To our knowledge, this study is the first to assess association of tuberculosis with risk factors among cancer patients in Thai population. In our study, we found an incidence rate of 421 to 848 per 100,000 per year, depending on the group of cancer types. The main 
Table 1. Demographic Characteristics and Post-Cancer TB Diagnosis Status of Cancer Patients

\begin{tabular}{|c|c|c|c|c|c|c|}
\hline \multirow[b]{2}{*}{ Characteristics } & \multirow[b]{2}{*}{ Cancer Patients (n) } & \multicolumn{2}{|c|}{ Cancer patients with no TB } & \multicolumn{2}{|c|}{ Post-Cancer TB diagnosis } & \multirow[b]{2}{*}{$P$-value } \\
\hline & & $\mathrm{n}$ & $\%$ & $\mathrm{n}$ & $\%$ & \\
\hline Age & & & & & & 0.99 \\
\hline$<=50$ & 10,789 & 10,664 & 98.86 & 125 & 1.16 & \\
\hline $51-60$ & 9,972 & 9,859 & 98.87 & 113 & 1.13 & \\
\hline $61-70$ & 10,695 & 10,569 & 98.82 & 126 & 1.18 & \\
\hline$>70$ & 9,492 & 9,384 & 98.86 & 108 & 1.14 & \\
\hline Total & 40,948 & 40,476 & 98.85 & 472 & 1.15 & \\
\hline Median & 60 & 60 & & $60(17,89)$ & & \\
\hline (Min, Max) & $(10,89)$ & $(10,87)$ & & $58.94(14.70)$ & & \\
\hline Mean & 58.92 & 58.92 & & & & \\
\hline (SD) & $(15.15)$ & $(15.15)$ & & & & \\
\hline Sex & & & & & & $<0.001$ \\
\hline Male & 21,014 & 20,718 & 98.59 & 296 & 1.41 & \\
\hline Female & 19,934 & 19,758 & 99.12 & 176 & 0.88 & \\
\hline Total & 40,948 & 40,476 & 98.85 & 472 & 1.15 & \\
\hline
\end{tabular}

findings showed that the major factors associated with higher incidence of TB included aging, male gender, lung cancer, hematopoietic, lymphoma, and digestive organ cancers. Advanced age changes the immune system and, with time, leads to increased vulnerability to infectious diseases (Nikolich-Žugich, 2018). Immunosenescence affects various cell types in the bone marrow and the thymus, mature lymphocytes in the peripheral blood and secondary lymphatic organs, and also elements of the innate immune system (Weiskopf et al., 2009). Therefore, elderly cancer patients, which were prone to immunosuppression, experienced higher incidences of TB compared to the young age group. Considering gender, male is indeed a risk factors for TB. Biological mechanisms may actually account for a significant part of this difference between male and female susceptibility to TB (Lienhardt et al., 2005; Neyrolles and Quintana-Murci, 2009). Females naturally present more effective immune responses and pathogen resistance, such as vaccination and infection, than males. Gender differences in other risk factors of TB may indirectly impact the male-to-female ratio of $\mathrm{TB}$, such as smoking, alcohol consumption and concurrent HIV infection. Males could have differing levels of M. tuberculosis exposure compared to females because of differences in social roles and activities. Males tend to travel, have direct contact, and spend time in confined places that increase transmission of infectious diseases (Nhamoyebonde and Leslie, 2014)

Cancer patients are increased risk of developing TB. In particular, systemic infections can be a risk when the host immune responses fail to uncontrol bacterial replication and active disease ensues (Delogu et al., 2013). Healthy individuals can harbor latent TB infections for their entire lives, but in around 5 to 15 percent of infected individuals, TB disease can be reactivated (Vynnycky and Fine, 1997; Kiazyk and Ball, 2017). Immunocompromised individuals are at increased risk of reactivation of latent TB infections, which includes patients hematological malignancies and

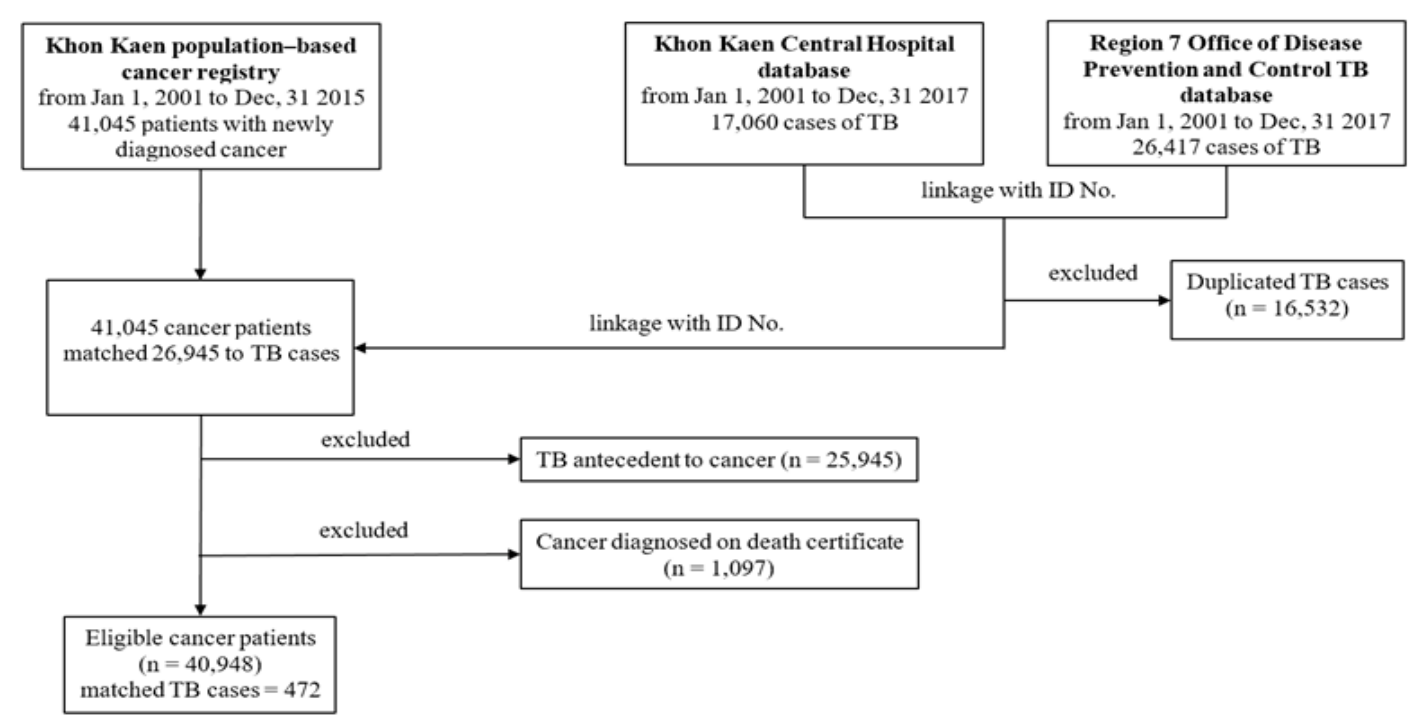

Figure 1. Data Flow Chart 
Table 2. Clinical Characteristics and Post-Cancer TB Diagnosis Status of Cancer Patients

Cancer patients with no TB Post-Cancer TB diagnosis

\begin{tabular}{|c|c|c|c|c|c|c|}
\hline Characteristics & Cancer Patients (n) & $\mathrm{n}$ & $\%$ & $\mathrm{n}$ & $\%$ & $\mathrm{p}$-value \\
\hline Cancer type & & & & & & $<0.001$ \\
\hline Liver and Bile Duct CA & 12,948 & 12,888 & 99.54 & 60 & 0.46 & \\
\hline Female organ CA & 3,701 & 3,671 & 99.19 & 30 & 0.81 & \\
\hline Breast CA & 3,647 & 3,619 & 99.23 & 28 & 0.77 & \\
\hline Colorectal CA & 3,224 & 3,190 & 98.95 & 34 & 1.0 .5 & \\
\hline Lung CA (no histology) & 2,656 & 2,599 & 97.85 & 57 & 2.15 & \\
\hline Head and neck CA & 2,376 & 2,340 & 98.48 & 36 & 1.52 & \\
\hline Hematopoietic & 1,844 & 1,790 & 97.07 & 54 & 2.93 & \\
\hline Unknown primary CA & 1,591 & 1,566 & 98.43 & 25 & 1.57 & \\
\hline Skin CA & 1,308 & 1,285 & 98.24 & 23 & 1.76 & \\
\hline Lung CA (histology) & 1,253 & 1,231 & 98.24 & 22 & 1.76 & \\
\hline Digestive organ CA & 1,207 & 1,194 & 98.92 & 13 & 1.08 & \\
\hline Lymphoma & 1,114 & 1,080 & 96.95 & 34 & 3.05 & \\
\hline Thyroid CA & 963 & 954 & 99.07 & 9 & 0.93 & \\
\hline Urinary tract $\mathrm{CA}$ & 902 & 885 & 98.12 & 17 & 1.88 & \\
\hline Male organ CA & 852 & 836 & 98.12 & 16 & 1.88 & \\
\hline Other CA & 831 & 822 & 98.92 & 9 & 1.08 & \\
\hline Brain CA & 531 & 526 & 99.06 & 5 & 0.94 & \\
\hline Total & 40,948 & 40,476 & 98.85 & 472 & 1.15 & \\
\hline \multicolumn{7}{|l|}{ Cancer stage (by extent) } \\
\hline Localized & 2,483 & 2,442 & 98.35 & 41 & 1.65 & \\
\hline Regional & 5,834 & 5,767 & 98.85 & 67 & 1.15 & \\
\hline Distant & 8,066 & 7,991 & 99.07 & 75 & 0.93 & \\
\hline Not applicable & 2,381 & 2,309 & 96.98 & 72 & 3.02 & \\
\hline Unknown stage & 22,184 & 21,967 & 99.02 & 217 & 0.98 & \\
\hline Total & 40,948 & 40,476 & 98.85 & 472 & 1.15 & \\
\hline Cancer treatment & & & & & & 0.018 \\
\hline No treatment & 22,361 & 22,121 & 98.93 & 240 & 1.07 & \\
\hline Only SURG & 6,685 & 6,600 & 98.73 & 85 & 1.27 & \\
\hline Only CMT & 2,942 & 2,888 & 98.16 & 54 & 1.84 & \\
\hline Only RT & 1,841 & 1,822 & 98.97 & 19 & 1.03 & \\
\hline SURG+CMT & 4,148 & 4,110 & 99.08 & 38 & 0.92 & \\
\hline SURG+RT & 1,304 & 1,290 & 98.93 & 14 & 1.07 & \\
\hline $\mathrm{CMT}+\mathrm{RT}$ & 824 & 813 & 98.67 & 11 & 1.33 & \\
\hline SURG+CMT+RT & 843 & 832 & 98.7 & 11 & 1.3 & \\
\hline Total & 40,948 & 40,476 & 98.85 & 472 & 1.15 & \\
\hline
\end{tabular}

patients undergoing immunosuppressant cancer therapies such as chemotherapy (Dobler et al., 2017). Our study found that certain cancer types were associated with higher risk of TB, including lung cancer, lymphoma, digestive organ cancer, hematopoietic and urinary tract cancer. For lung cancer, it is possible for misdiagnosis due to mimic finding on the chest. The first case of coexistent TB and lung cancer was reported by Bayle in 1810 . (Bayle, 1810; Singh et al., 2009; Christopoulos et al., 2014) It has been well documented that lung inflammation and fibrosis from TB could induce genetic damage, which may increase the risk of lung cancer. (Liang et al., 2009) Reverse causality is also possible, as occult lung cancer may cause TB infection and provoke reactivation of latent TB by weakening the local immunity. (Society, 2000; Wu et al., 2011).

For lymphoma, it is likely that TB infection affected bone marrow suppression. Our results were consistent with several studies in which lymphoma increased the risk of the development of active TB. (Cheng et al., 2017; Ganzel et al., 2019) The incidence of TB in Non-Hodgkin lymphoma (NHL) patients is 35 times higher than in the general population. Furthermore, interleukin 10 (IL-10), which is increased in patients with NHL, has been implicated in the reactivation of TB. The serum concentration of IL-10 has been found to be higher in 
Table 3. Incidence Rate Ratios (IRRs) for TB by Risk Factor among Cancer Patients Based on Multiple Poisson Regression $(\mathrm{n}=9,733)$

\begin{tabular}{|c|c|c|c|c|c|c|c|c|}
\hline Variables & $\begin{array}{c}\text { Cancer } \\
\text { Patients (n) }\end{array}$ & $\begin{array}{l}\text { TB cases } \\
(\mathrm{n})\end{array}$ & $\begin{array}{l}\text { Person time } \\
\text { (person-year) }\end{array}$ & $\begin{array}{c}\text { IR } \\
(100,000 \\
\text { patients per year })\end{array}$ & $\begin{array}{l}\text { Crude } \\
\text { IRR }\end{array}$ & $\begin{array}{l}\text { Adjusted } \\
\text { IRR }\end{array}$ & $\begin{array}{c}95 \% \mathrm{CI} \\
\text { adjusted IRR }\end{array}$ & $\mathrm{p}$-value \\
\hline Age & & & & & & & & $<0.001$ \\
\hline$<=50$ & 2,711 & 52 & 11,470 & 453.31 & 1 & 1 & & \\
\hline $51-60$ & 2,177 & 52 & 5,220 & 996.94 & 2.2 & 1.63 & $1.09-2.43$ & \\
\hline $61-70$ & 2,571 & 51 & 4,760 & 1070.52 & 2.36 & 1.52 & $1.01-2.27$ & \\
\hline$>70$ & 2,334 & 51 & 2,830 & 1799.79 & 3.97 & 2.36 & $1.56-3.55$ & \\
\hline Total & 9,733 & 206 & 24,280 & & & & & \\
\hline Gender & & & & & & & & $<0.001$ \\
\hline Female & 5,796 & 150 & 13,110 & 426.91 & 1 & 1 & & \\
\hline Male & 3,937 & 56 & 11,170 & 1343.19 & 3.15 & 1.87 & $1.36-2.59$ & \\
\hline Total & 9,733 & 206 & 24,280 & & & & & \\
\hline Cancer type* & & & & & & & & $<0.001$ \\
\hline Thyroid CA & 954 & 9 & 6,900 & 130.53 & 1 & 1 & & \\
\hline Lung CA** & 1,231 & 22 & 1,460 & 1506.11 & 11.54 & 5.48 & $2.16-13.91$ & \\
\hline Lymphoma & 1,080 & 34 & 3,770 & 901.36 & 6.91 & 4.15 & $1.62-10.61$ & \\
\hline Hematopoietic & 1,790 & 54 & 5,250 & 1027.95 & 7.88 & 3.76 & $1.46-9.73$ & \\
\hline Digestive organ CA & 1,194 & 13 & 1,430 & 908.34 & 6.96 & 3.9 & $1.51-10.12$ & \\
\hline Urinary tract CA & 885 & 17 & 3,170 & 535.85 & 4.11 & 2.27 & $0.91-5.67$ & \\
\hline Lung $\mathrm{CA}^{* * *}$ & 2,599 & 57 & 2,300 & 2478.22 & 18.99 & 6.22 & $2.57-15.04$ & \\
\hline Total & 9,733 & 206 & 24,280 & & & & & \\
\hline Cancer stage (by extent) & & & & & & & & 0.476 \\
\hline Local & 225 & 2 & 1,390 & 143.78 & 1 & 1 & & \\
\hline Regional & 843 & 14 & 1,910 & 733.02 & 5.1 & 2.59 & $0.58-12.54$ & \\
\hline Distant & 2,196 & 29 & 2,310 & 1257.79 & 8.75 & 2.69 & $0.63-11.54$ & \\
\hline Not applicable & 2,033 & 67 & 6,340 & 1056.48 & 7.35 & 2.83 & $0.65-12.32$ & \\
\hline Unknown & 4,436 & 94 & 12,340 & 761.96 & 5.3 & 2.24 & $0.54-9.28$ & \\
\hline Total & 9,733 & 206 & 24,280 & & & & & \\
\hline \multicolumn{9}{|l|}{ Cancer Treatment } \\
\hline Only SURG & 1,253 & 19 & 5,180 & 367.08 & 1 & 1 & & 0.05 \\
\hline No treatment & 5,184 & 120 & 7,550 & 1587.26 & 4.32 & 1.63 & $0.84-3.05$ & \\
\hline Only CMT & 1,782 & 44 & 5,470 & 804.51 & 2.19 & 0.94 & $0.47-1.88$ & \\
\hline Only RT & 459 & 7 & 960 & 730.09 & 1.99 & 1.12 & $0.43-2.89$ & \\
\hline SURG+CMT & 346 & 4 & 1,220 & 328.76 & 0.9 & 0.47 & $0.16-1.40$ & \\
\hline SURG+RT & 401 & 4 & 2,890 & 138.44 & 0.38 & 0.91 & $0.28-2.97$ & \\
\hline $\mathrm{CMT}+\mathrm{RT}$ & 258 & 7 & 850 & 822.05 & 2.24 & 0.97 & $0.37-2.55$ & \\
\hline SURG+CMT+RT & 50 & 1 & 160 & 613.13 & 1.67 & 1 & $0.13-7.63$ & \\
\hline Total & 9,733 & 206 & 24,280 & & & & & \\
\hline
\end{tabular}

Remark; IRR, Incidence rate ratio; 95\% CI , 95\% confidence interval; 1, Reference group; SURG, surgery; CMT, chemotherapy; RT, Radiotherapy; *, Included only 7 cancer types in the multiple poisson regression; **, Lung cancer with histological confirmation; ***, Lung cancer without histological confirmation

patients with NHL, which may lead to decreased cell mediated immunity resulting in activation of latent TB (Hashmi et al., 2017) Hematopoietic cancer was related to TB disease developing. In this study, the cancer type with the highest proportion of patients receiving no treatment was hematopoietic cancer $(59.1 \%$; data not shown). Therefore, TB infection may affect the disease itself. Other studies have shown the relative risk of TB disease in hematologic malignancy patients is $2-40$ times as compared with the general population (Anibarro and Pena, 2014). Not only the disease itself but also the robust chemotherapy or aggressive therapeutic procedures used to suppress the immune system in hematological cancer patients may lead to TB in these patients and the hematological cancer patients bone marrow or stem cell recipients also tended to have $\mathrm{TB}$ as well $(\mathrm{Wu}$ et al., 2011). In addition, digestive organ cancer has been shown to be a risk factors for the development of TB in our study. It was consistent with Kim et al., which found that immune suppression by the tumor increased vulnerability to reactivation of TB (Kim et al., 2008). Several studies from Taiwan and Korea have also 
identified gastric cancer as a significant risk factor for TB (Fang et al., 2015; Jung et al., 2016). The digestive organ in this study included esophagus, stomach, small intestine and other ill-defined digestive organs. As these organs are important to nutrition, digestive organ dysfunction can lead to malnutrition and opportunistic infection. Interestingly, higher incidence of TB was associated with urinary tract cancer in this study. The incidence rate ratios among urinary tract cancer patients in this study (2.27) were similar to those in bladder cancer patients in Taiwan (1.38) (Wu et al., 2011).

In our study, we used surgery as a reference group in analysis of the effects of treatment, as it was least likely to affect the immune system when comparing to other modalities and to avoid selection bias of using no treatment. Although chemotherapy is an immunosuppressant, we found no significant difference for risk of TB according to cancer treatment. This result may have been due to a limitation of this study, in which the curative and palliative roles of treatment could not be differentiated.

These findings suggest increased TB screening may be necessary in high risk cancer patients (lung cancer, lymphoma, hematopoietic and digestive organ cancer) and older age group ( $>70$ year). According to published literature, other types of cancer, such as head and neck cancer and breast cancer, should also be considered for increased TB screening (Cheng et al., 2017). This study adopted the retrospective cohort study for the burden of cancer with TB demonstration. Population-based of cancer registry and TB in the same define population was analyzed for achieved goal. Furthermore, the study period that is long enough to illustrate the change in its trends.

This study reports the incidence rate of TB among cancer patients in a TB endemic area. Risk factors associated with TB among cancer patients included older age group, males, and certain types of cancer, especially lung cancer, lymphoma, hematopoietic cancer, and digestive organ cancer. Increased caution and awareness may be necessary among cancer patients to reduce spread and morbidity. We recommend TB surveillance and prevention plans with emphasis on cancer patients. Increased screening for latent TB infections may also be necessary during cancer treatment.

\section{Acknowledgements}

The authors would like to thank staff from the Cancer Unit at the Faculty of Medicine of Srinagarind Hospital at Khon Kaen University; Khon Kaen Central Hospital; and the Bureau of Tuberculosis, Department of Disease Control, Ministry of Public Health for providing the data and the Epidemiology Unit of the Faculty of Medicine at the Prince of Songkla University for technical support during the study.

\section{References}

American Thoracic Society (2000). Targeted tuberculin testing and treatment of latent tuberculosis infection. Am J Respir Crit Care Med, 161, S221-47.

Anibarro L, Pena A (2014). Tuberculosis in patients with haematological malignancies. Mediterr $J$ Hematol Infect Dis, 6, e2014026

Bureau of Tuberculosis, Department of Disease Control, Ministry of Public Health (2017). Nation strategy planning 2017-2021. Bureau of Tuberculosis, Department of Disease of Control, Bangkok.

Bayle GL (1810). Recherches sur la phthisie pulmonaire: Ouvrage lu à la Société de la faculté de médecine de Paris, dans diverses séances, en 1809 et 1810 . Gabon, French.

Cheng MP, Chakra CNA, Yansouni CP, et al (2017). Risk of active tuberculosis in patients with cancer: A systematic review and metaanalysis. Clin Infect Dis, 64, 634-44.

Choi IJ, Kim YW, Lee HS, et al (2015). Risk factors for TB in patients with early gastric cancer: Is gastrectomy a significant risk factor for TB?. Chest, 148, 774-83.

Christopoulos A, Saif MW, Sarris EG, Syrigos KN (2014). Epidemiology of active tuberculosis in lung cancer patients: A systematic review. Clin Respir J, 8, 375-81.

Delogu G, Sali M, Fadda G(2013). The biology of mycobacterium tuberculosis infection. Mediterr J Hematol Infect Dis, 5, e2013070.

Dobler CC, Cheung K, Nguyen J, Martin A (2017). Risk of tuberculosis in patients with solid cancers and haematological malignancies: A systematic review and meta-analysis. Eur Respir J, 50, e1700157.

Fang WL, Hung YP, Liu CJ, et al (2015). Incidence of and risk factors for tuberculosis (TB) in gastric cancer patients in an area endemic for TB: A nationwide population-based matched cohort study. Medicine, 94, e2163.

Ganzel C, Silverman B, Chemtob D, Ben Shoham A, Wiener-Well Y (2019). The risk of tuberculosis in cancer patients is greatest in lymphoma and myelodysplastic syndrome/myeloproliferative neoplasm: A large populationbased cohort study. Leuk Lymphoma, 60, 720-5.

Hashmi HRT, Mishra R, Niazi M, Venkatram S, Diaz-Fuentes $\mathrm{G}$ (2017). An unusual triad of hemophagocytic syndrome, lymphoma and tuberculosis in a non-HIV patient. Am J Case Rep, 18, 739-45.

Huang SF, Li CP, Feng JY, Chao Y, Su WJ (2011). Increased risk of tuberculosis after gastrectomy and chemotherapy in gastric cancer: A 7-year cohort study. Gastric Cancer, 14, 257-65.

Jung IY, Kim MH, Jeong WY, et al (2016). Treatment outcomes of patients treated for pulmonary tuberculosis after undergoing gastrectomy. Tohoku J Exp Med, 240, 281-6.

Kiazyk S, Ball T B (2017). Latent tuberculosis infection: An overview. CA Communicable Dis Rep, 43, 62-66.

Kim HR, Hwang SS, Ro YK, et al (2008). Solid-organ malignancy as a risk factor for tuberculosis. Respirology, 13, 413-9.

Liang HY, Li XL, Yu XS, et al (2009). Facts and fiction of the relationship between preexisting tuberculosis and lung cancer risk: A systematic review. Int J Cancer, 125, 2936-44.

Lienhardt C, Fielding K, Sillah JS, et al (2005). Investigation of the risk factors for tuberculosis: A case-control study in three countries in West Africa. Int J Epidemiol, 34, 914-23.

Neyrolles O, Quintana-Murci L (2009). Sexual inequality in tuberculosis. PLoS Med, 6, e1000199.

Nhamoyebonde S, Leslie A (2014). Biological differences between the sexes and susceptibility to tuberculosis. J Infect Dis, 209, S100-6.

Nikolich-Žugich J (2018). The twilight of immunity: Emerging concepts in aging of the immune system. Nat Immunol, 19, 10-9.

Singh VK, Chandra S, Kumar S, et al (2009). A common medical error: Lung cancer misdiagnosed as sputum negative tuberculosis. Asian Pac J Cancer Prev, 10, 335-8. 
Teng C J, Huon L K, Zheng Y M, et al (2019). Increased risk of tuberculosis in oral cancer patients in an endemic area: a nationwide population-based study. Clin Oral Investigat, 23, 4223-31.

Vynnycky E, Fine P E (1997). The natural history of tuberculosis: the implications of age-dependent risks of disease and the role of reinfection. Epidemiol Infect, 119, 183-201.

Weiskopf D, Weinberger B, Grubeck-Loebenstein B (2009). The aging of the immune system. Transpl Int, 22, 1041-50.

Wu CY, Hu HY, Pu CY, et al (2011). Aerodigestive tract, lung and haematological cancers are risk factors for tuberculosis: An 8-year population-based study. Int J Tuberc Lung Dis, 15, 125-30.

World Health Organization (2018). Global tuberculosis report. Available: https://www.who.int/tb/publications/ global_report/en.

This work is licensed under a Creative Commons AttributionNon Commercial 4.0 International License. 